\title{
Star formation in the central kpc region of NGC1614
}

R. Herrero-Illana ${ }^{a}$, M.Á. Pérez-Torres ${ }^{a, b, c}$, A. Alberdi ${ }^{a}$, A. Alonso-Herrero ${ }^{d}$, L. Colina $^{e}$, A. Efstathiou ${ }^{f}$, L. Hernández-García ${ }^{a}$, D. Miralles-Caballero ${ }^{g}$, P. Väisänen ${ }^{h, i}$, C. Packham $^{j}$, V. Rajpaul ${ }^{k}$, and A. Zijlstra ${ }^{l}$

${ }^{a}$ Instituto de Astrofísica de Andalucía - CSIC, PO Box 3004, 18008, Granada, Spain

${ }^{b}$ Centro de Estudios de Física del Cosmos de Aragón (CEFCA), E-44001, Teruel, Spain

${ }^{c}$ Dpto. de Física Teórica, Universidad de Zaragoza, E-50009, Zaragoza, Spain

${ }^{d}$ Instituto de Física de Cantabria, CSIC-Universidad de Cantabria, 39005, Santander, Spain

${ }^{e}$ Centro de Astrobiología (INTA-CSIC), Ctra. de Torrejón a Ajalvir, km 4, 28850, Torrejón de Ardoz, Madrid, Spain

${ }^{f}$ School of Sciencies, European University Cyprus, Diogenes Street, Engomi, 1516, Nicosia, Cyprus

${ }^{g}$ Instituto de Física Teórica, Universidad Autónoma de Madrid, 28049, Madrid, Spain

${ }^{h}$ South African Astronomical Observatory, P.O. Box 9, Observatory 7935, Cape Town, South Africa

${ }^{i}$ Southern African Large Telescope, P.O. Box 9, Observatory 7935, Cape Town, South Africa

${ }^{j}$ Department of Physics and Astronomy, University of Texas at San Antonio, One UTSA Circle, San Antonio, TX 78249, USA

${ }^{k}$ Department of Physics, University of Oxford, Denys Wilkinson Building, Keble Road, Oxford OX1 3RH, UK

${ }^{l}$ Jodrell Bank Centre for Astrophysics, University of Manchester, Manchester M13 9PL, UK

E-mail: herrero@iaa.es, torres@iaa.es, antxon@iaa.es,

aalonsolifca.unican.es, colina@cab.inta-csic.es,

A.Efstathioudeuc.ac.cy, lorena@iaa.es, thorius@gmail.com,

petri@saao.ac.za, CHRIS.PACKHAM@UTSA.EDU,

Vinesh.Rajpaul@astro.ox.ac.uk, albert.zijlstra@manchester.ac.uk

The Luminous Infrared Galaxy NGC1614 hosts a prominent circumnuclear ring of star formation[1]. However, the nature of the dominant emitting mechanism in its central $\sim 100 \mathrm{pc}$ is still under debate. We present sub-arcsecond angular resolution radio, mid-infrared, Pa $\alpha$, optical, and X-ray observations of NGC 1614, aimed at studying in detail both the circumnuclear ring and the nuclear region. We conclude that there is no need to invoke an AGN to explain the observed bolometric properties of the galaxy. This study is detailed in Herrero-Illana et al. 2012 [4].

12th European VLBI Network Symposium and Users Meeting,

7-10 October 2014

Cagliari, Italy 


\section{A multi-wavelength comparison}

Our main aim is to shed light on the AGN/SB nature of the nuclear region of NGC1614. We discuss the striking morphological similarities between the radio and mid-IR images, which suggest a common origin for both emission mechanisms.

The most conspicuous feature is the prominent mid-IR emission of the nucleus, $\mathrm{N}$, which contrasts with its rather faint emission at radio wavelengths (see Fig. 1). The regions to the northwest of the ring, $\mathrm{A}$ and $\mathrm{B}$, show a mid-IR/radio ratio below the average of the whole ring (R), while regions $\mathrm{C}$ and $\mathrm{D}$ show the opposite behavior.

The nuclear region, $\mathrm{N}$, shows a non-thermal spectral index of $\alpha \sim-1.80$ between 3.6 and $6.0 \mathrm{~cm}$. Such a steep spectral index seems to be at odds with an AGN origin, which typically present flat or even inverted indices [5]. This steep index, however, can be reconciled with the scenario of a compact starburst. The radio spectral index is the average value over region $\mathrm{N}$, of $\sim 90 \mathrm{pc}$ in radius, so in principle we cannot rule out completely the existence of a hidden AGN inside that region, as found in other LIRGs, e.g., in Arp 299-A [8]. However, even if there is an AGN, its radio luminosity would not contribute more than $\sim 6 \%$ and $\sim 7.6 \%$ at 3.6 and $6.0 \mathrm{~cm}$, respectively [4]. Multi-wavelength diagnostic plots also locate NGC1614 in the starburst dominated region [7]. Radio VLBI observations would provide the final diagnostic to completely exclude the presence of an AGN.

\section{Spatially Resolved X-ray Emission}

We fitted the Chandra spectrum using four different models: (1) a pure thermal model (MEKAL), where the thermal emission is responsible for the bulk of the X-ray energy distribution; (2) an absorbed PL model, which corresponds to a non-thermal source representing an AGN; (3) a composite of a thermal plus an absorbed PL model (MEPL); and (4) a thermal model with tuned individual abundances (VMEKAL), which models the metal abundance pattern of type II SNe. Our best fit turned out to be the VMEKAL, giving support to the idea of a pure starburst model. This model finds abundances for Mg XI (1.36 keV), Si XIII (1.85 keV), and S XV (2.4 keV). The corresponding luminosity in the soft band for this model is $\log L_{(0.5-2 \mathrm{keV})}=40.78 \mathrm{erg} \mathrm{s}^{-1}$.

\section{Radio thermal and non-thermal components}

Massive stars and their associated HII regions are responsible for the thermal free-free radio emission, while SNe and SNRs account for the non-thermal synchrotron radio emission Using extinction-corrected Pa $\alpha$ measurements, we inferred the amount of each type of radio emission. We found that:

$$
S_{\text {thermal }}=1.076 \times 10^{13} \times F(\mathrm{~Pa} \alpha) v^{-0.1} .
$$

The ratio of thermal free-free to synchrotron radio emission can be used as an indicator of the SB age of each region in the circumnuclear ring of NGC1614. The ratios of thermal to nonthermal radio emission for regions $\mathrm{A}$ and $\mathrm{B}$ are about $\sim 0.5$, while those of regions $\mathrm{C}$ and $\mathrm{D}$ of 1.1 and 1.2, respectively. The ratios above (and the free-free thermal continuum luminosities) can be well explained if the emission in regions $\mathrm{C}$ and $\mathrm{D}$ come from instantaneous bursts with ages 

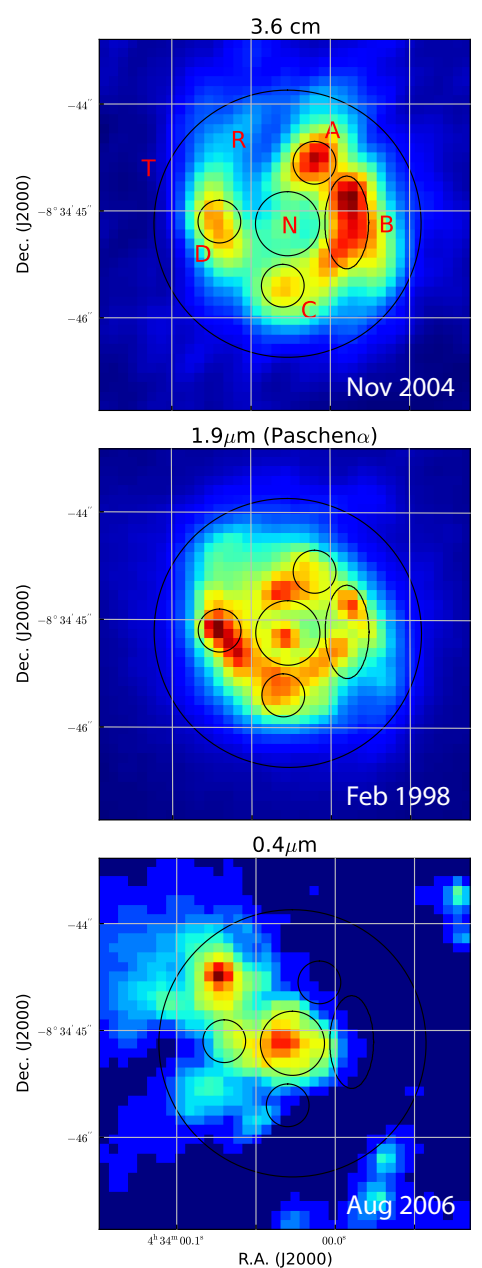

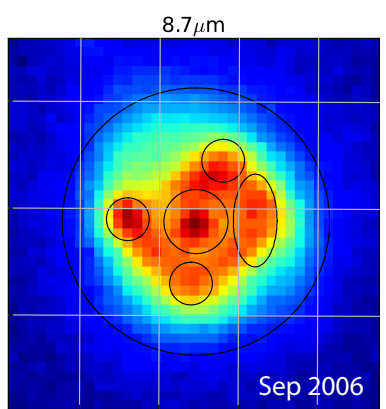

$3.3 \mu \mathrm{m}(\mathrm{PAH})$

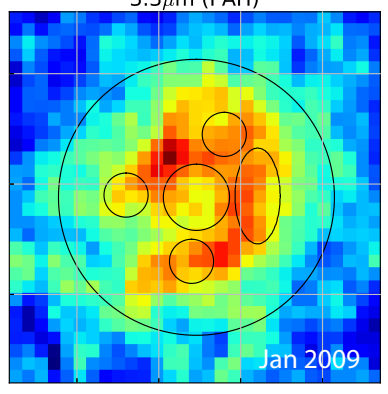

$0.8 \mu \mathrm{m}$

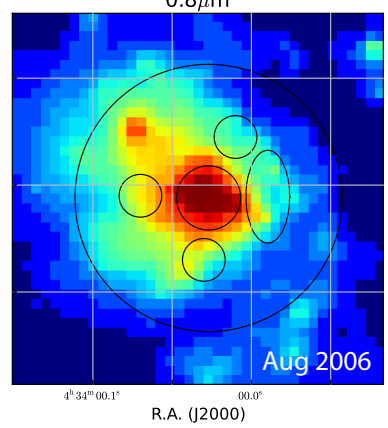

Figure 1: Multi-wavelength comparison of the nuclear region of NGC1614. Based on the radio emission, we defined seven regions to compare the images, including the nuclear region $(\mathrm{N})$, the whole ring $(\mathrm{R})$, and the total area (T). Note the close similarity in the star formation ring and the contrast in the nuclear region between the radio and mid-IR wavelengths.

$<5.5 \mathrm{Myr}$, where SNe have only recently started to explode. On the other hand, the emission from regions A and B would come from slightly older $(\sim 8 \mathrm{Myr})$ bursts. Since the thermal free-free emission is directly proportional to the $\mathrm{Pa} \alpha$ flux, the decomposed images would indicate that the radio emission from the nuclear region is dominated by thermal free-free emission, which in turn suggests it is powered by a starburst, rather than an AGN.

\section{SED model fit}

We modeled NGC1614 SED using an exponentially decaying starburst model, by combining pure SB models from [3] and models from the host galaxies [2]. Our model (see Fig. 2) yields a bolometric luminosity of $10^{11.39} L_{\odot}$, an initial SFR of $85.1 M_{\odot} \mathrm{yr}^{-1}$, a core collapse supernova rate of $0.43 \mathrm{SNyr}^{-1}$, and an ionizing photon flux of $3.47 \times 10^{54} \mathrm{~s}^{-1}$. The contribution of the AGN to the total bolometric luminosity, if any, would be in any case $<10 \%$. 


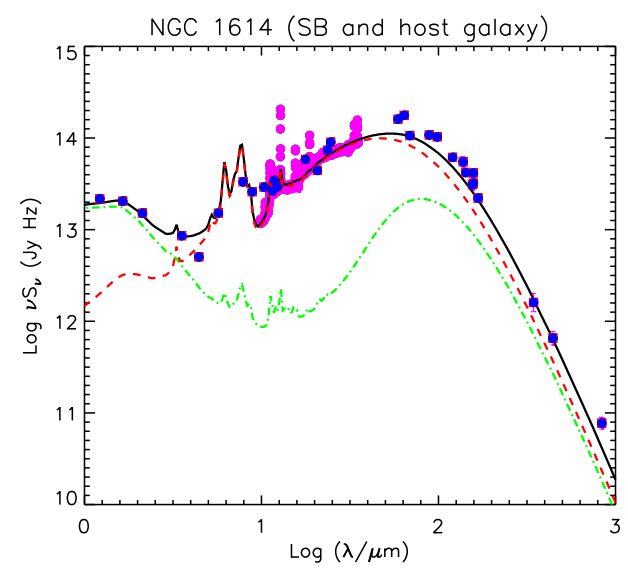

Figure 2: NGC1614 SED fitting. Photometric points are plotted in blue and pink. Lines show the overall fit (solid black), the starburst contribution (dashed red), and the host galaxy contribution (dot-dashed green). There is no need to include an AGN component.

\section{References}

[1] A. Alonso-Herrero, C. W. Engelbracht, M. J. Rieke, G. H. Rieke, and A. C. Quillen, NGC 1614: A Laboratory for Starburst Evolution, ApJ 546 (2001), 952-965.

[2] G. Bruzual and S. Charlot, Stellar population synthesis at the resolution of 2003, MNRAS 344 (2003), 1000-1028.

[3] A. Efstathiou and R. Siebenmorgen, Starburst and cirrus models for submillimeter galaxies, A\&A 502 (2009), 541-548.

[4] R. Herrero-Illana, M. Á. Pérez-Torres, A. Alonso-Herrero, A. Alberdi, L. Colina, A. Efstathiou, L. Hernández-García, D. Miralles-Caballero, P. Väisänen, C. C. Packham, V. Rajpaul, and A. A. Zijlstra, A Multi-wavelength View of the Central Kiloparsec Region in the Luminous Infrared Galaxy NGC 1614, ApJ 786 (2014), 156.

[5] N. M. Nagar, H. Falcke, and A. S. Wilson, Radio sources in low-luminosity active galactic nuclei. IV. Radio luminosity function, importance of jet power, and radio properties of the complete Palomar sample, A\&A 435 (2005), 521-543.

[6] D. E. Perez-Olea and L. Colina, X-Ray Emission of Active Galactic Nuclei with Circumnuclear Star-forming Rings: NGC 1097 and NGC 7469, ApJ 468 (1996), 191.

[7] M. A. Pérez-Torres, A. Alberdi, C. Romero-Cañizales, and M. Bondi, Serendipitous discovery of the long-sought active galactic nucleus in Arp 299-A, A\&A 519 (2010), L5+.

\section{Acknowledgments}

The European VLBI Network (EVN) is a joint facility of European, Chinese, South African, and other radio astronomy institutes funded by their national research councils. RHI, MPT, and AA acknowledge support from the Spanish MINECO through grant AYA2012-38491-C02-02. The research leading to these results has received funding from the European Commission Seventh Framework Programme (FP/2007-2013) under grant agreement No 283393 (RadioNet3). 